\title{
Lanthanides to Quantum Dots Resonance Energy Transfer in Time-Resolved FluoroImmunoAssays and
} Luminescence Microscopy

Loïc J. Charbonnière, ${ }^{\dagger *}$ Niko Hildebrandt, ${ }^{\text {+*}^{*}}$ Raymond F. Ziessel, ${ }^{\dagger}$ and Hans-Gerd Löhmannsröben

\section{Supplementary Information (7 pages including this one)}

\section{Table of contents}

Table T1: Luminescence decay times, $\tau_{\mathrm{D}}{ }^{\mathrm{a}}{ }^{\mathrm{a}}$ and lanthanide centered quantum yields, $\Phi_{\mathrm{Ln}}{ }^{\mathrm{b}}$ for EuL-Strep, TbL-Strep and $\mathrm{Eu}(\mathrm{TBP})$-Strep in different buffering media.

Page S2

Table T2: Overlap integrals $J_{\lambda}$ and Förster radii $R_{0}$ of the donor-acceptor pairs with Biot-QD as acceptor .

Page S3

Figure S1: Determination of the Ln-Strep labeling ratio. Left: UV-Vis spectra of Ln-Strep, Strep, pure Ln complex and a linear combination of Strep and Ln complex. Right: MALDI-TOF spectra of streptavidin and its three lanthanide labeled bioconjugates. Number of Ln complexes per streptavidin monomer displayed on top of the peaks.

Figure S2: Influence of azide concentration on TbL-NHS ([TbL-NHS $]=2 \mathrm{x}$ $\left.10^{-6} \mathrm{M}\right)$. Azide concentration is $0,0.01,0.05,0.1,0.25$ and $0.5 \%$ from top to bottom, respectively.

Figure S3: Influence of azide concentration on TbL-Strep ([TbL-Strep $]=4 \mathrm{x}$ $\left.10^{-8} \mathrm{M}\right)$. Azide concentration is $0,0.01,0.05,0.1,0.25$ and $0.5 \%$ from top to bottom, respectively. The two-exponential decay behaviour becomes obvious at a concentration of $0.05 \%$ azide.

Figure S4 : Relative luminescence intensities of EuL-Strep (full symbols) and TbL-Strep (hollow symbols) after $20 \mathrm{~min}$. (squares) and 5 hours (dots) incubation time as a function of [BSA]. [TbL-Strep] $=1 \times 10^{-9}$ to $3.4 \times 10^{-10} \mathrm{M}$ and $[$ EuL-Strep $]=3 \times 10^{-9}$ to $1 \times 10^{-9} \mathrm{M}$.

Page S4

Page S5

Page S5

Figure S5: TbL control experiment for dynamic energy transfer.

Page S6

Page S6

Figure S6: EuL control experiment for dynamic energy transfer.

Page S7

Figure S7: $\mathrm{Eu}(\mathrm{TBP})$ control experiment for dynamic energy transfer.

Page S7

Figure S8: Eu(TBP)-APC control experiment for dynamic energy transfer.

Page S7 
Table T1: Luminescence decay times, $\tau_{\mathrm{D}}{ }^{\mathrm{a}}{ }^{\mathrm{a}}$ and lanthanide centered quantum yields, $\Phi_{\mathrm{Ln}}{ }^{\mathrm{b}}$, for EuL-Strep, TbLStrep and Eu(TBP)-Strep in different buffering media.

\begin{tabular}{|c|c|c|c|c|c|c|}
\hline & $\tau_{\mathrm{D}}$ & EuL-Strep & $\tau_{\mathrm{D}}$ & $\Phi_{\mathrm{Ln}}$ & $\begin{array}{r}\mathrm{Eu}(\mathrm{TBP} \\
\tau_{\mathrm{D} 1}(\mu \mathrm{s}, \mathrm{rel} . \\
\tau_{\mathrm{D} 2}(\mu \mathrm{s}, \mathrm{rel} .\end{array}$ & $\begin{array}{l}\text { rep } \\
\qquad \Phi_{\mathrm{Ln}}\end{array}$ \\
\hline Water & 680 & 0.26 & 1480 & 0.49 & $\begin{array}{l}850(55) \\
320(45)\end{array}$ & 0.34 \\
\hline Borate buffer, $\mathrm{pH} 8.3$ & 650 & 0.25 & 1220 & 0.40 & $\begin{array}{l}650(65) \\
230(35)\end{array}$ & 0.30 \\
\hline $\begin{array}{c}\text { Borate buffer, } \mathrm{pH} 8.3+2 \% \\
\text { BSA }\end{array}$ & 650 & 0.25 & 1220 & 0.40 & $\begin{array}{l}690(63) \\
230(37)\end{array}$ & 0.31 \\
\hline $\begin{array}{c}\text { Borate buffer, } \mathrm{pH} 8.3+2 \% \\
\text { BSA }+0.05 \% \mathrm{NaN}_{3}\end{array}$ & 680 & 0.26 & 690 & 0.22 & $\begin{array}{l}1050(67) \\
230(33)\end{array}$ & 0.45 \\
\hline Water $+0.5 \mathrm{M} \mathrm{KF}$ & 1400 & 0.54 & 2200 & 0.72 & $1080(100)$ & 0.63 \\
\hline $\begin{array}{c}\text { Borate buffer, } \mathrm{pH} 8.3+2 \% \\
\mathrm{BSA}+0.5 \mathrm{M} \mathrm{KF}\end{array}$ & 1400 & 0.54 & 1940 & 0.65 & $\begin{array}{c}1040(100) \\
-\end{array}$ & 0.61 \\
\hline $\begin{array}{c}\text { Borate buffer, } \mathrm{pH} 8.3+2 \% \\
\mathrm{BSA}+0.5 \mathrm{M} \mathrm{KF}+0.05 \% \mathrm{NaN}_{3}\end{array}$ & 1400 & 0.54 & 1450 & 0.48 & $\begin{array}{c}1100(100) \\
-\end{array}$ & 0.65 \\
\hline
\end{tabular}

a) $\left(\lambda_{\text {exc }}=315\right.$ or $337 \mathrm{~nm}, \lambda_{\mathrm{em}}=(545 \pm 5) \mathrm{nm}$ for $\mathrm{Tb}$ and $(618 \pm 5) \mathrm{nm}$ for Eu) estimated exp. uncertainty $\pm 5 \%$.

b) estimated exp. uncertainty $\pm 10 \%$. 
Table T2: Overlap integrals $J_{\lambda}$ and Förster radii $R_{0}$ of the donor-acceptor pairs with Biot-QD as acceptor .

\begin{tabular}{|c|c|c|c|c|}
\hline & & $\begin{array}{l}\text { borate buffer } \\
\text { pH } 8.3\end{array}$ & $\begin{array}{c}\text { borate buffer } \\
\mathrm{pH} 8.3+2 \% \text { BSA }\end{array}$ & $\begin{array}{c}\text { borate buffer } \\
\mathrm{pH} 8.3+2 \% \text { BSA }+ \\
0.5 \mathrm{M} \mathrm{KF} \\
\end{array}$ \\
\hline \multirow[b]{2}{*}{ TbL-Strep } & $\begin{array}{c}J_{\lambda} \text { in } \\
\mathrm{M}^{-1} \mathrm{~cm}^{-1} \mathrm{~nm}^{4}\end{array}$ & $1.3 \times 10^{17}$ & $1.3 \times 10^{17}$ & $1.3 \times 10^{17}$ \\
\hline & $R_{0}(\AA)$ & $96 \pm 2$ & $96 \pm 2$ & $104 \pm 2$ \\
\hline \multirow{2}{*}{ EuL-Strep } & $\begin{array}{c}J_{\lambda} \text { in } \\
\mathrm{M}^{-1} \mathrm{~cm}^{-1} \mathrm{~nm}^{4}\end{array}$ & $9.5 \times 10^{16}$ & $9.5 \times 10^{16}$ & $9.5 \times 10^{16}$ \\
\hline & $R_{0}(\AA)$ & $84 \pm 2$ & $84 \pm 2$ & $96 \pm 2$ \\
\hline \multirow{2}{*}{ Eu(TBP)-Strep } & $\begin{array}{c}J_{\lambda} \text { in } \\
\mathrm{M}^{-1} \mathrm{~cm}^{-1} \mathrm{~nm}^{4}\end{array}$ & $7.8 \times 10^{16}$ & $7.8 \times 10^{16}$ & $6.2 \times 10^{16}$ \\
\hline & $R_{0}(\AA)$ & $84 \pm 2$ & $85 \pm 2$ & $91 \pm 2$ \\
\hline
\end{tabular}



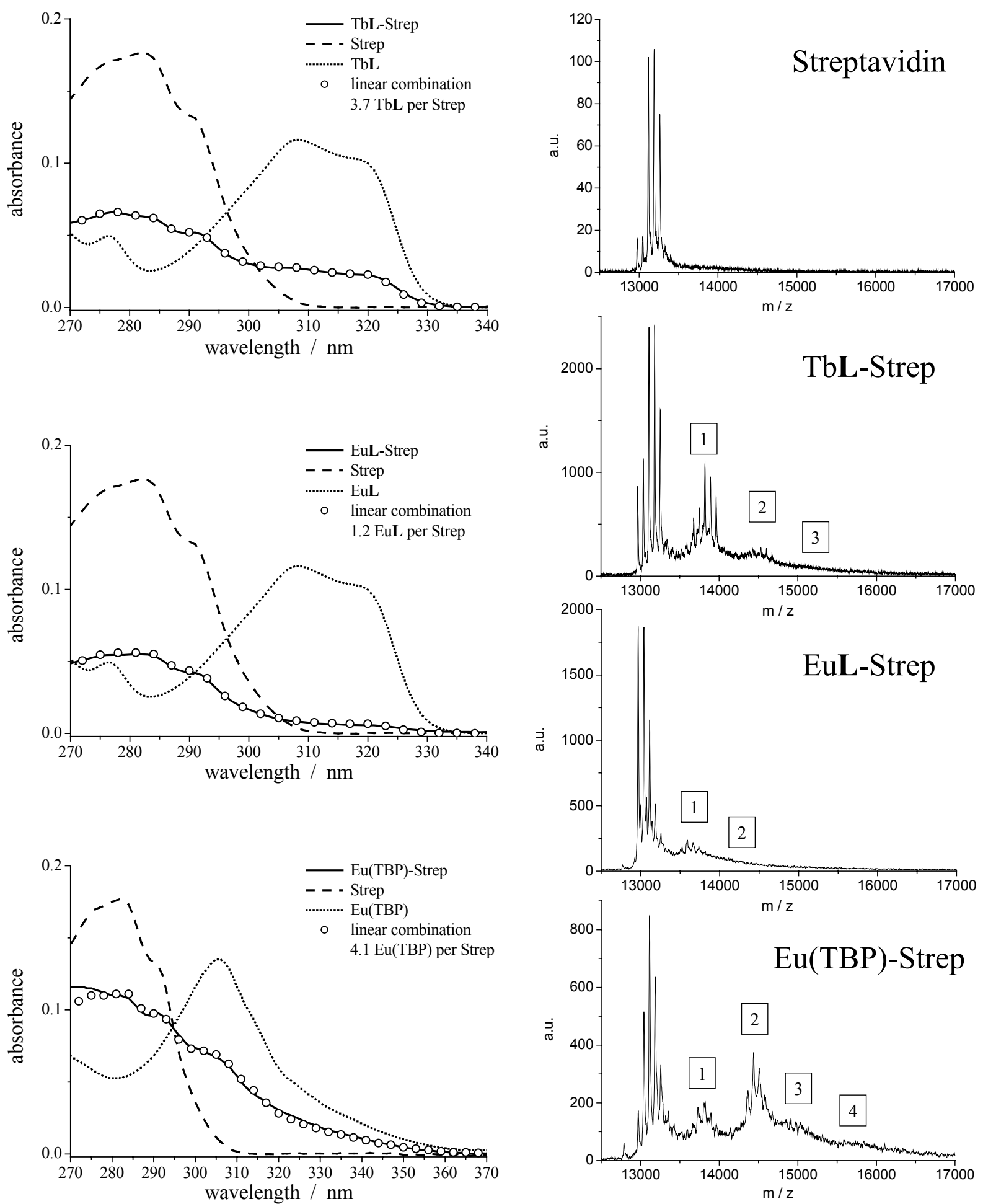

Figure S1: Determination of the Ln-Strep labeling ratio. Left: UV-Vis spectra of Ln-Strep, Strep, pure Ln complex and a linear combination of Strep and Ln complex. Right: MALDI-TOF spectra of streptavidin and its three lanthanide labeled bioconjugates. Number of Ln complexes per streptavidin monomer displayed on top of the peaks. 


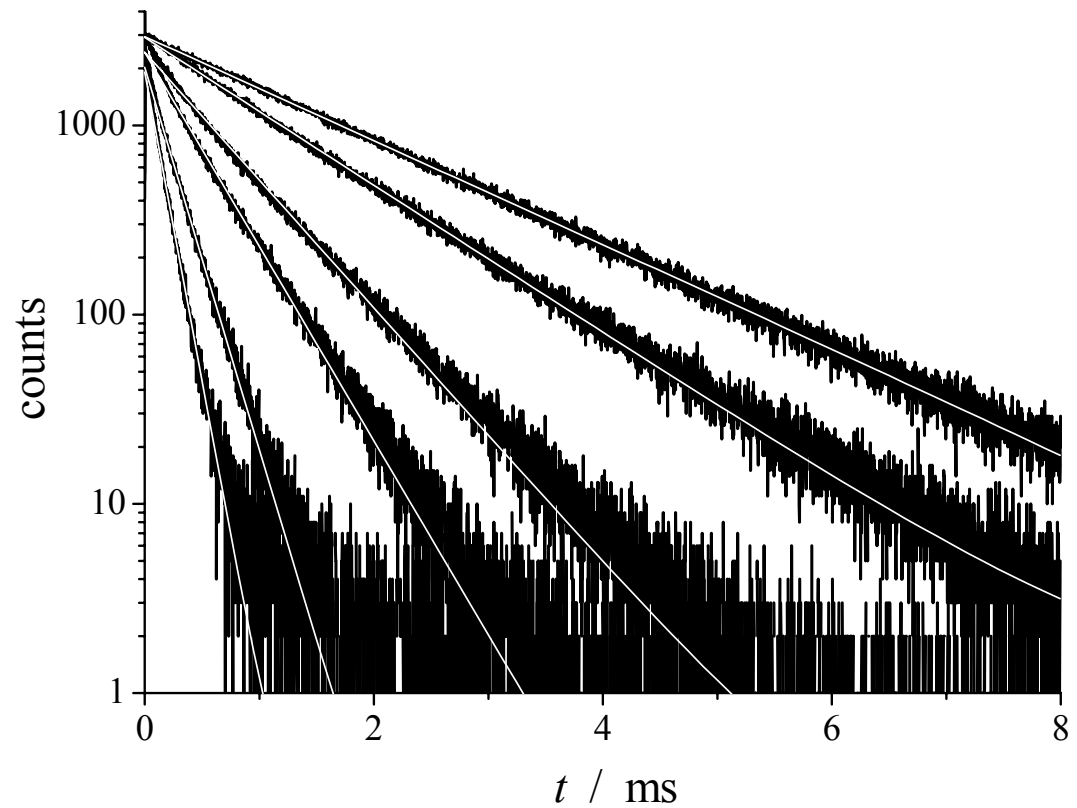

Figure S2: Influence of azide concentration on TbL-NHS ([TbL-NHS $\left.]=2 \times 10^{-6} \mathrm{M}\right)$. Azide concentration is 0 , $0.01,0.05,0.1,0.25$ and $0.5 \%$ from top to bottom, respectively.

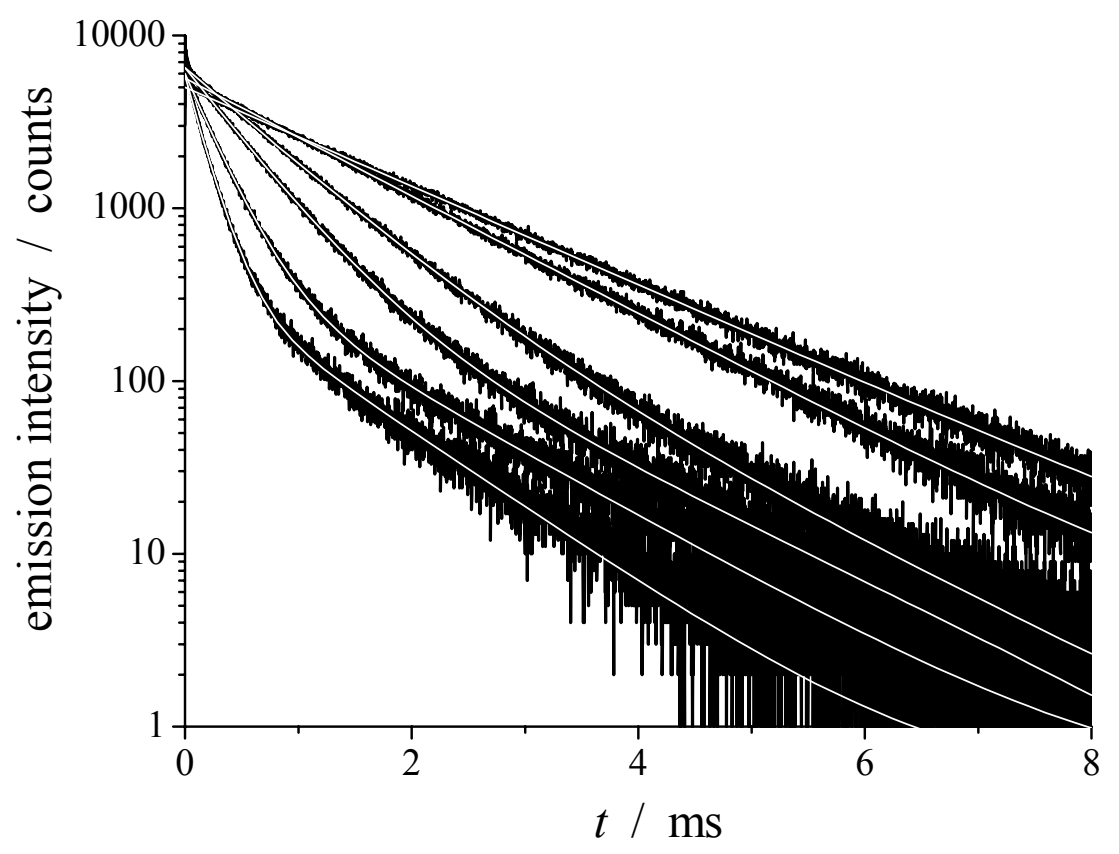

Figure S3: Influence of azide concentration on TbL-Strep $\left([\mathrm{TbL}-\mathrm{Strep}]=4 \times 10^{-8} \mathrm{M}\right)$. Azide concentration is 0 , $0.01,0.05,0.1,0.25$ and $0.5 \%$ from top to bottom, respectively. The two-exponential decay behaviour becomes obvious at a concentration of $0.05 \%$ azide. 


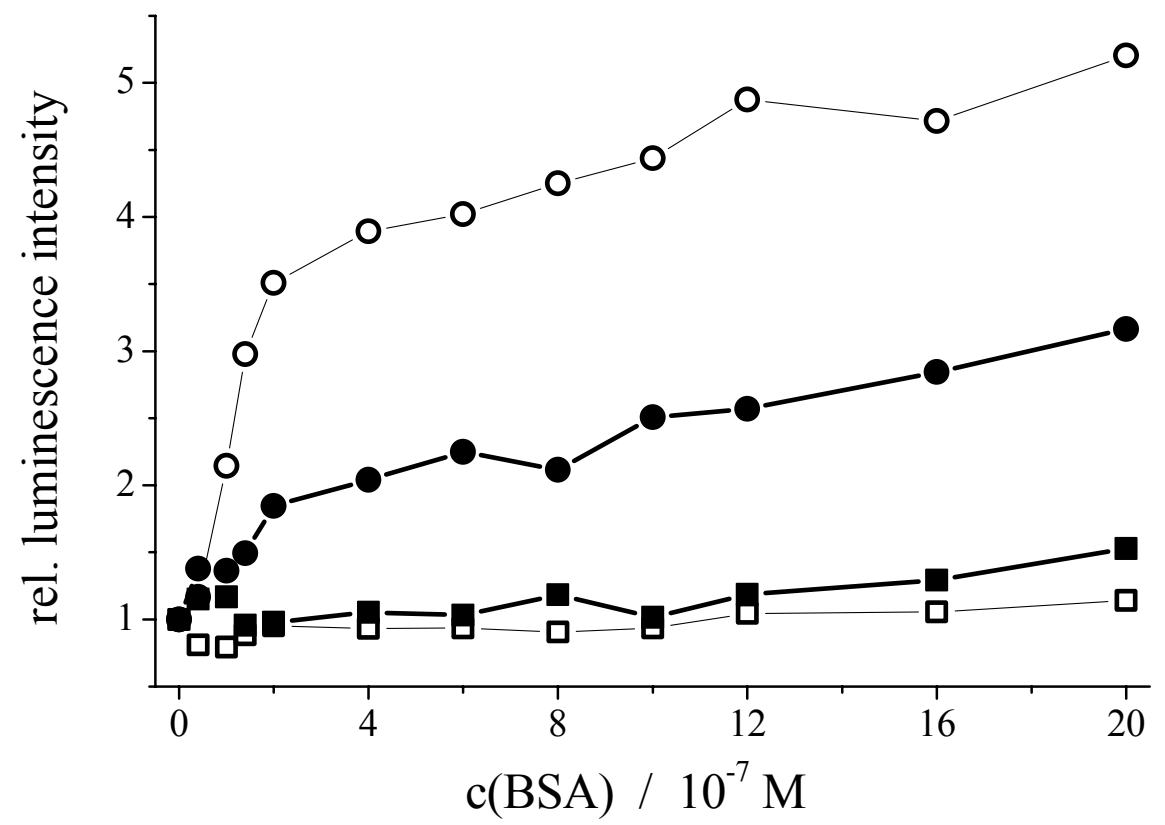

Figure S4 : Relative luminescence intensities of EuL-Strep (full symbols) and TbL-Strep (hollow symbols) after 20 min. (squares) and 5 hours (dots) incubation time as a function of [BSA]. [TbL-Strep] $=1 \times 10^{-9}$ to $3.4 \times 10^{-}$ ${ }^{10} \mathrm{M}$ and $\left[\right.$ EuL-Strep] $=3 \times 10^{-9}$ to $1 \times 10^{-9} \mathrm{M}$.

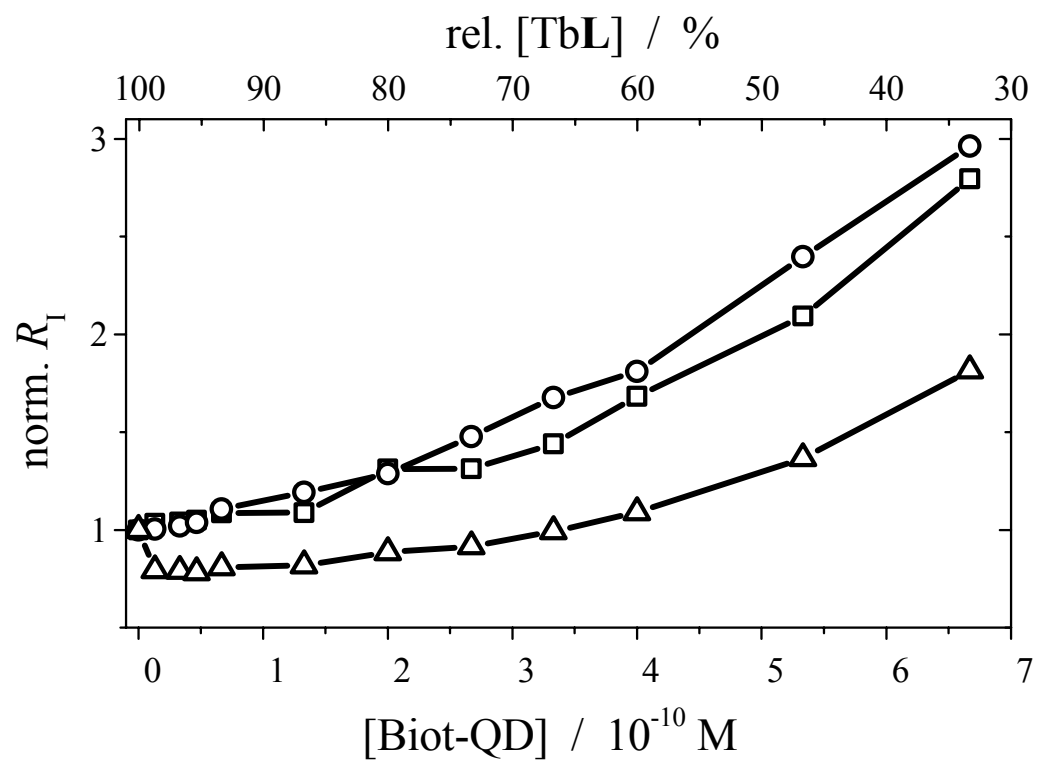

Figure S5: TbL control experiment for dynamic energy transfer. 
rel. $[\mathrm{EuL}]$ / \%

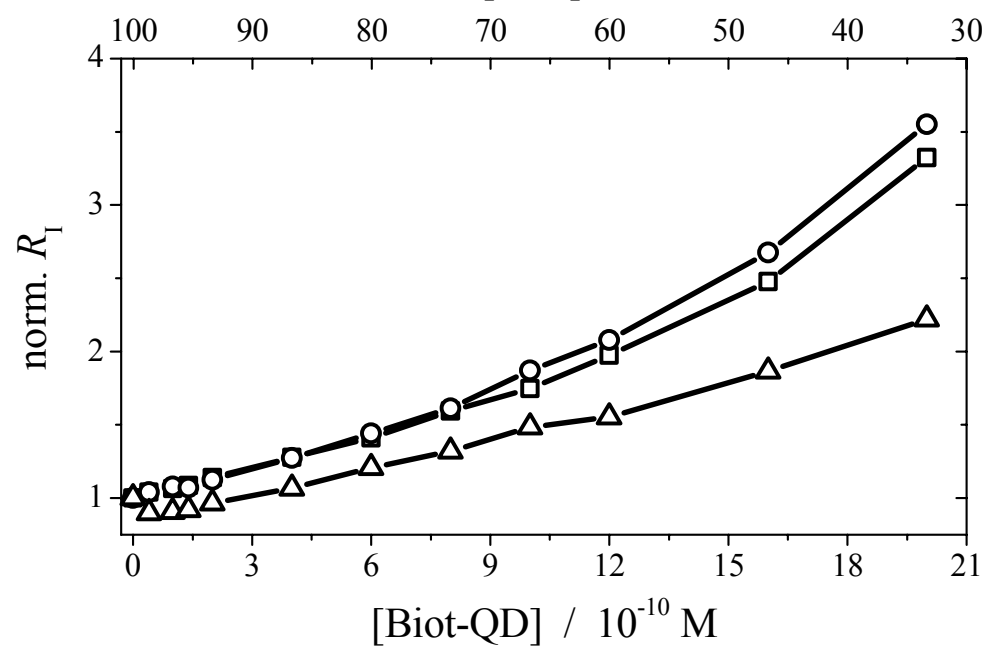

Figure S6: EuL control experiment for dynamic energy transfer.

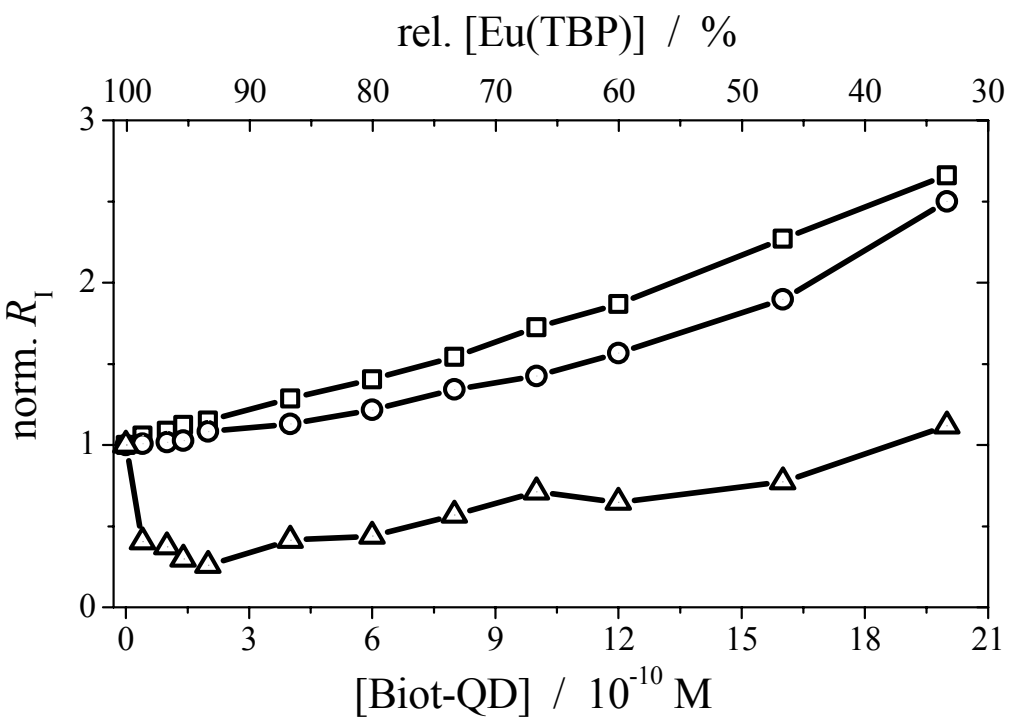

Figure S7: Eu(TBP) control experiment for dynamic energy transfer.

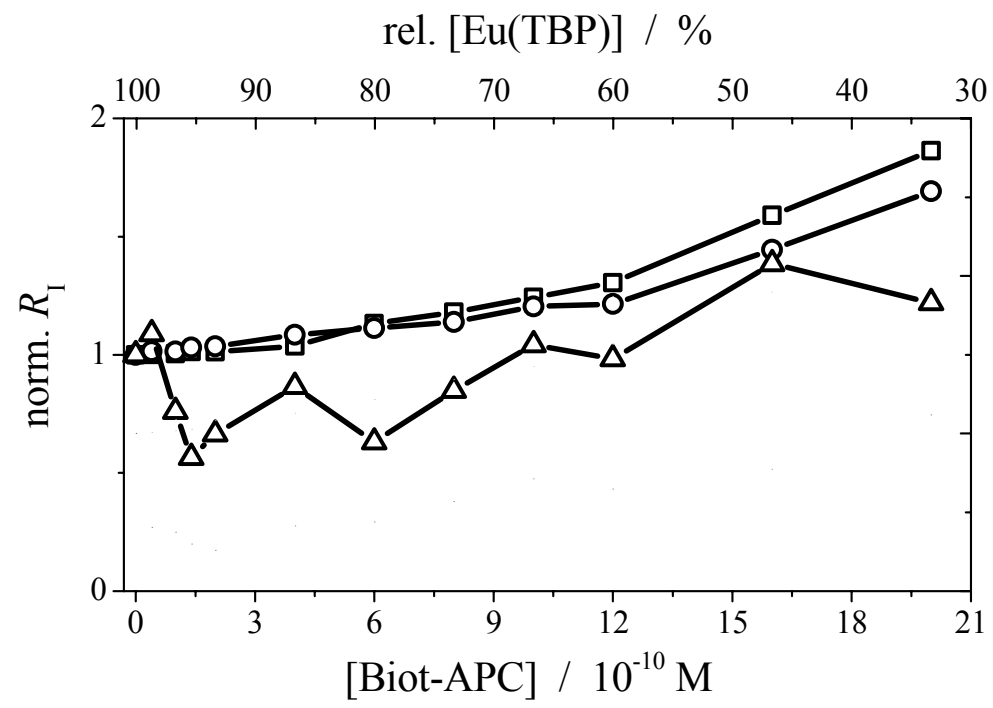

Figure S8: Eu(TBP)-APC control experiment for dynamic energy transfer. 